\title{
Imaging of HIV-associated parotid gland enlargement
}

\section{G Rozendaal, MB ChB}

Department of Radiology, Pretoria Academic Hospital

Parotid gland enlargement may be the presenting clinical manifestation of systemic HIV infection in children as well as adults. ${ }^{1}$ Comprehensive salivary gland evaluation is therefore mandatory in patients with uni- or bilateral parotid gland swelling. This article highlights the imaging techniques (ultrasound, computed tomography (CT), magnetic resonance imaging (MRI)) used in the investigation of salivary gland enlargement and identifies the specific radiological signs found in HIV infection. The presence of diffuse, multiple cysts in one or both enlarged parotid glands represents benign lymphoepithelial proliferation with cyst formation and is found in systemic HIV infection. Concurrent hyperplastic cervical and intraparotid lymphadenopathy may also be seen., ${ }^{2,3}$

The aetiology of parotid gland enlargement is extensive and it may be the presenting clinical manifestation of systemic HIV infection in children as well as adults. Comprehensive salivary gland evaluation is therefore mandatory in patients with uni- or bilateral parotid gland swelling.

The specific radiological signs of parotid gland enlargement in HIV infection are discussed here.

Usual findings during ultrasound examination would be an enlarged parotid gland with diffuse, multiple, small $(1-4 \mathrm{~mm})$ to medium-sized (5-8 mm) hypoechoic or anechoic (cystic) areas. Less commonly, large anehoic (cystic) areas almost totally replacing the gland are seen. Focal intra-parotid solid lesions and extra-parotid lymph nodes may also be identified (Fig. 1).

CT images show enlarged parotid glands with diffuse, multiple, variably sized, intraglandular cysts with low attenuation values (10 - 18 Hounsfield units). The presence of solid focal intra-parotid mass lesions may suggest lymphadenopathy or concurrent Kaposi's sarcoma. Enlarged peri-parotid and cervical lymph nodes may also be demonstrated (Fig. 2).

MRI supports the CT findings noted above. The high water content of the intra-parotid cysts is responsible for the different signal intensities obtained, with T1-weighted images having low or mixed signal intensity. The cysts have intermediate signal intensity on proton density-weighted images and bright signal intensity T2-weighted images (Fig. 3).

The histological features include a diffuse lymphocytic infiltration of the parotid gland with formation of lymphoepithelial cysts and follicular hyperplasia of intra- and peri-parotid lymph nodes ${ }^{4}$ (Fig. 4).

Ultrasound, CT and MRI demonstrate diffuse, multiple cysts in one or both enlarged parotid glands with associated hyperplastic cervical and intra-parotid lymphadenopathy seen mostly on CT and MRI images. On histological examination these lesions represent benign lymphoepithelial proliferation with cyst formation.
The pathogenesis of benign lymphoepithelial cysts remains unclear although a reactive lymphoid tissue infiltration of salivary glands associated with the hyperactivity of the immune system in HIV has been suggested. Salivary gland duct obstruction secondary to the exuberant cellular process could also account for the cyst formation.

Ultrasound is not as accurate as CT or MRI, but offers a rapid, simple, relatively inexpensive imaging technique to ascertain the nature of salivary gland pathology.

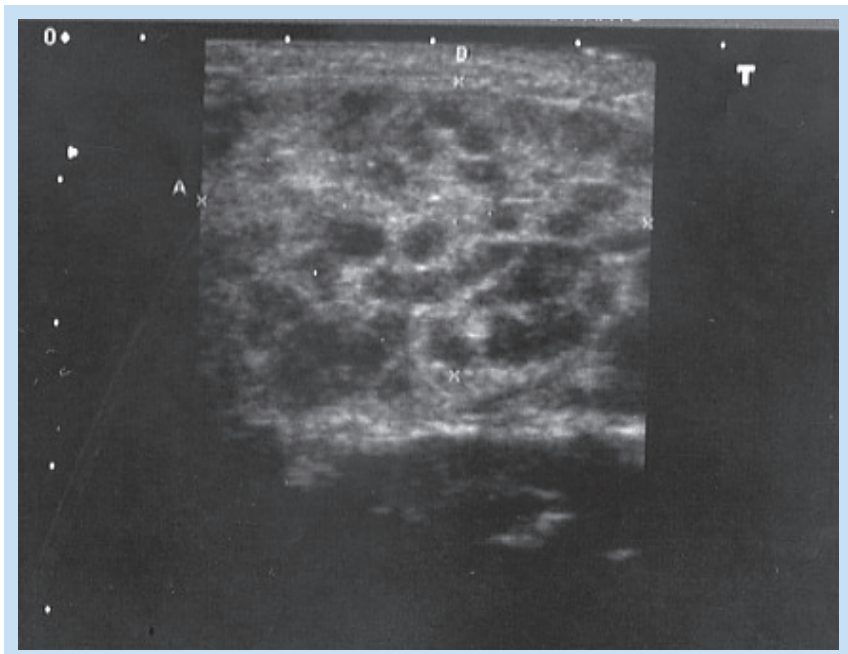

Fig. 1. Ultrasound: Multiple sonolucent areas representing lymphoepithelial cysts.

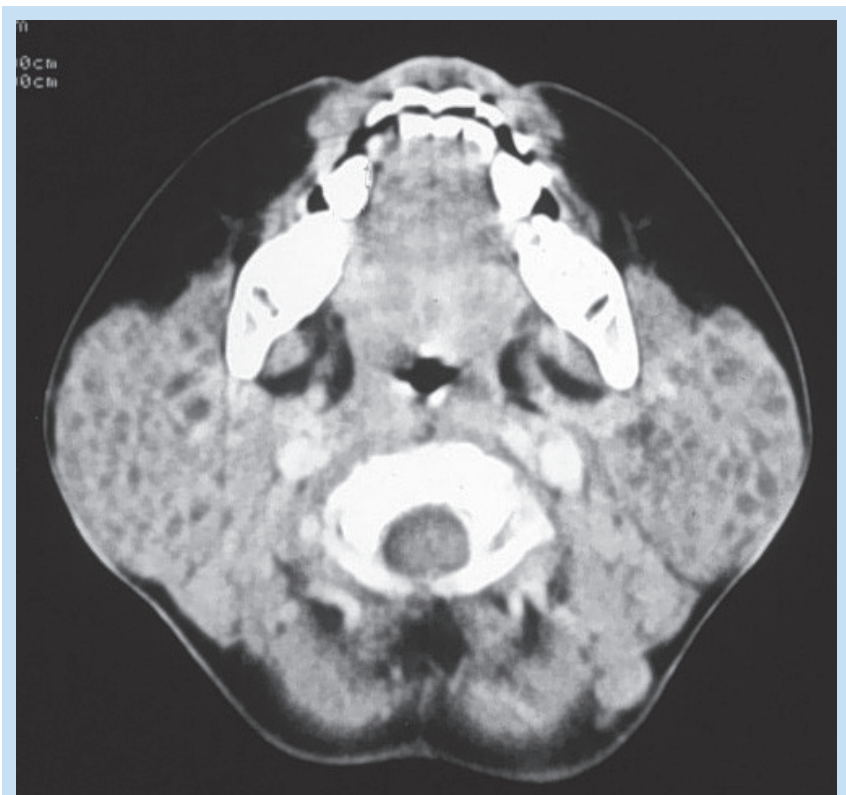

Fig. 2. Axial CT (post contrast): Enlarged bilateral parotid glands with multiple small cysts and posterior cervical lymphadenopathy. 


\section{PICTORIAL INTERLUDE}

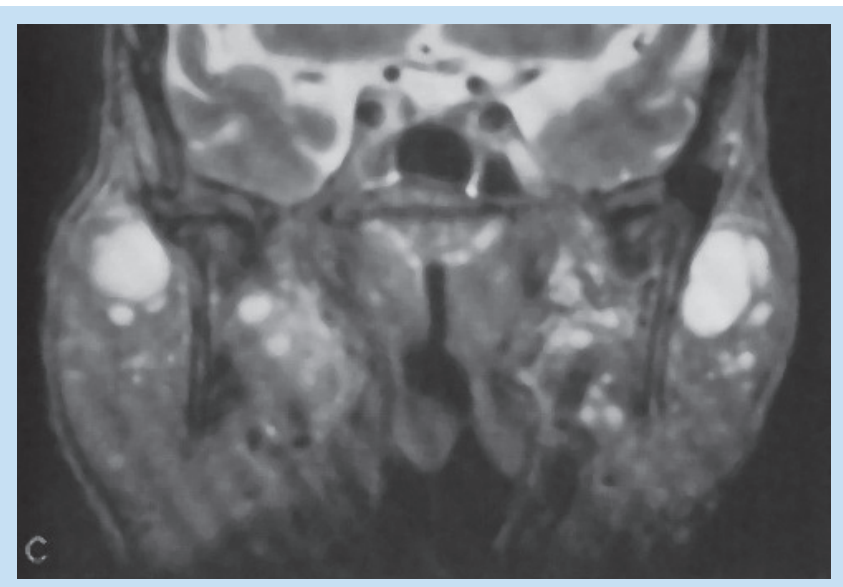

Fig. 3. MRI (T2-weighted image): Multiple variable sized cysts in both parotid glands.

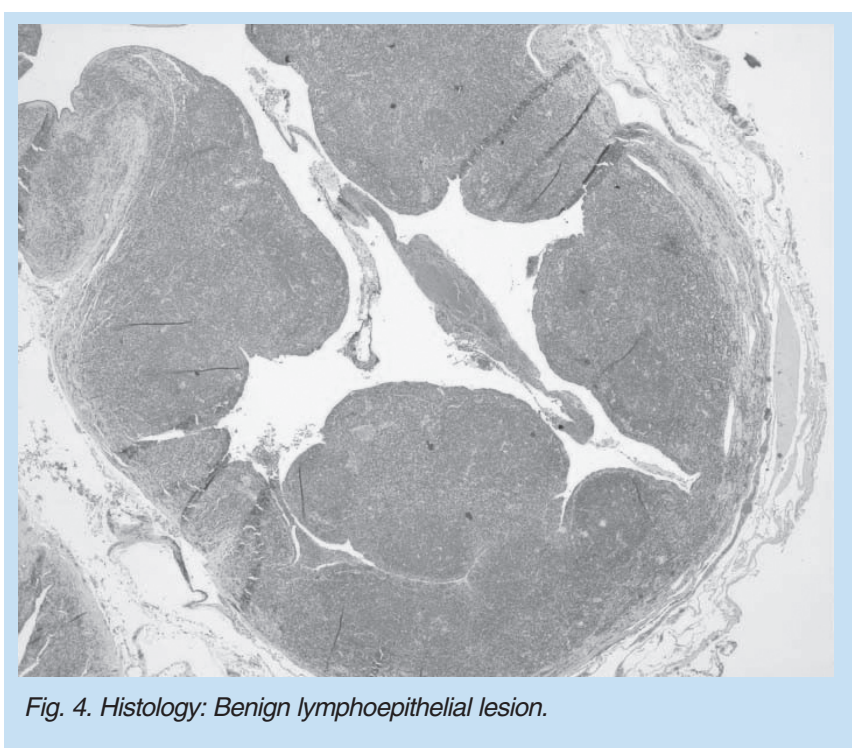

MRI demonstrates the key features of multi-cystic disease more clearly than CT scan.

\section{Conclusion}

Current imaging techniques used in visualising HIV-associated parotid gland enlargement include ultrasound, CT and MRI. The characteristic radiological picture identifies diffuse, multiple, small cysts in one or both enlarged parotid glands which, on histological examination, represent benign lymphoepithelial proliferation with cyst formation. Concurrent hyperplastic cervical and intra-parotid lymphadenopathy may also be seen.

Although ultrasound offers rapid and inexpensive imaging, CT and MRI demonstrate the pathological findings more clearly.

1. Chapnik JS, Noyek AM, Berris B, et al. Parotid gland enlargement in HIV infection: clinical/imaging findings. J Otolaryngol 1990; 19:189-194.

2. Kirshenbaum KJ, Nadimpalli SR, Friedman M, Kirshenbaum GL, Cavallino RP. Benign lymphoepithelial parotid tumors in AIDS patients: CT and MRI findings in nine cases. Am J Neuroradiol 1991; 12:271274.

3. Trunkel DE, Loury M, Fox C, Goins M, Johns M. Bilateral parotid enlargement in HIV-seropositive patients. Laryngoscope 1989; 99:590-595.

4. Rupp M, Chambersburg MD. Case 08-2002. HIV-associated salivary gland disease. http://www.afip. org/departments/OMaxPath

5. Department of Radiology, Chaing Mai University. Pediatric Radiology Case 2. http://www.medicine cmu.ac.th/dept/radiology

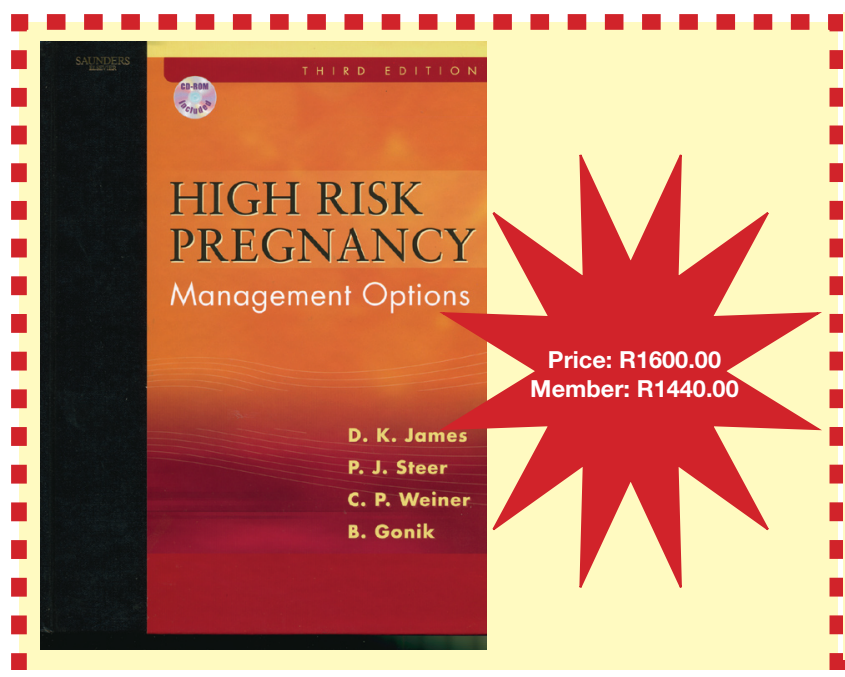

To order contact Carmen or Avril:

Tel: (02 1) 530-6520/27

Fax: (021) 531-4126/3539

email: carmena@hmpg.co.za or avrilm@hmpg.co.za

The South African Medical Association,

Health \& Medical Publishing Group

1-2 Lonsdale Building, Gardener Way, Pinelands, 7405.
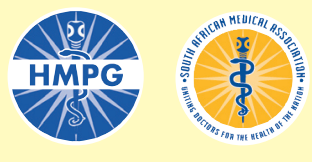